\title{
Fragmented QRS complexes are associated with left ventricular systolic and diastolic dysfunctions in patients with metabolic syndrome
}

\author{
Ender Oner ${ }^{1}$, Mehmet Erturk ${ }^{1}$, Ali Birant ${ }^{1}$, Ali Kemal Kalkan ${ }^{1}$, Fatih Uzun ${ }^{1}$, \\ Yalcin Avci ${ }^{2}$, Muhammet Gurdogan ${ }^{3}$, Hamdi Pusuroglu ${ }^{1}$, Aydin Yildirim ${ }^{1}$ \\ ${ }^{1}$ Department of Cardiology, Stanbul Mehmet Akif Ersoy Thoracic and Cardiovascular \\ Surgery Training and Research Hospital, Istanbul, Turkey \\ ${ }^{2}$ Clinic of Cardiology, Istanbul Bahcelievler State Hospital, Turkey \\ ${ }^{3}$ Clinic of Cardiology, Edirne State Hospital, Turkey
}

\begin{abstract}
Background: Metabolic syndrome (MetS) is found to be associated with deterioration of the left ventricular $(L V)$ systolic and diastolic functions. One of the factors for this impairment is myocardial fibrosis. Fragmented $Q R S$ ( $f Q R S)$ complexes are found to be associated with myocardial fibrosis. The aim of the study was to evaluate if the presence of $f Q R S$ on electrocardiogram (ECG) can detect pronounced impairment in the LV systolic and diastolic functions in MetS patients.
\end{abstract}

Methods: The study included 111 (mean age $47 \pm 9$, 49.5\% male) MetS patients and 96 (mean age $45 \pm 9,58.3 \%$ male) control subjects without MetS. ECG was evaluated for the presence of $f Q R S$. Each patient underwent conventional echocardiography and tissue Doppler imaging.

Results: Fragmented QRS was more common among MetSpatients (26.1\% vs. 14.6\%, $p=0.041)$. $M e t S$ was associated with subclinical LV systolic and LV diastolic dysfunctions. In subgroup analyses of MetS patients, the presence of $f Q R S$ on ECG had a higher E/E' ratio and lower E'velocity, indicating pronounced diastolic dysfunction, as well as lower isovolumic acceleration (IVA), indicating profound subclinical LV systolic dysfunction. E/E' ratio and IVA were independent predictors of $f Q R S$ presence in patients with MetS.

Conclusions: Fragmented QRS is more common among MetS patients compared to non-MetS patients. The presence of $f Q R S$ is associated with pronounced subclinical LV systolic and diastolic dysfunctions in MetS patients. (Cardiol J 2015; 22, 6: 691-698)

Key words: metabolic syndrome, fragmented QRS, isovolumic acceleration

\section{Introduction}

Metabolic syndrome (MetS) consists of a clustering of several metabolic and physiological risk factors, including hyperglycemia, hypertriglyceri- demia, lower high-density lipoprotein cholesterol (HDL-C), hypertension, and abdominal obesity [1]. MetS is diagnosed when three or more of these metabolic abnormalities are present in the same person, according to the National Cholesterol

Address for correspondence: Ender Oner, MD, Kardiyoloji Kliniği, Mehmet Akif Ersoy Göğüs Kalp ve Damar Cerrahisi, Eğitim ve Araştırma Hastanesi, İstasyon Mah, Turgut Özal Bulvarı No:11 Küçükçekmece, 34303, Istanbul, Turkey, tel: +90 21269220 00, fax: +90212 47194 94, e-mail: enderoner7@gmail.com 
Education Program Adult Treatment Panel III (NCEP ATP III) [2]. MetS has been shown to be associated with left ventricular (LV) systolic and diastolic dysfunctions [3-5]. The mechanism behind cardiac impairment in MetS is multifactorial, but one of the pivotal contributors is thought to be myocardial fibrosis [6, 7].

Fragmented QRS (fQRS) includes various RSR' patterns with different morphologies of the QRS complexes with or without the $Q$ wave on a resting 12-lead electrocardiogram (ECG). Various RSR' patterns include an additional $R$ wave ( $R$ ') or notching in the nadir of the $\mathrm{S}$ wave, or the presence of $>1$ R' (fragmentation) in 2 contiguous leads, corresponding to a major coronary artery territory [8]. Fragmented QRS has been shown to be a marker of myocardial fibrosis or scar tissue, and has been found to be associated with increased adverse cardiac events $[9,10]$. The aim of this study was to determine if $\mathrm{fQRS}$ is more common in MetS patients and if the presence of $\mathrm{fQRS}$ on ECG can detect pronounced impairment in LV systolic and diastolic functions in MetS patients.

\section{Methods}

This study was an observational case-control study.

\section{Study population}

The study was performed at the Mehmet Akif Ersoy Thoracic and Cardiovascular Surgery Training and Research Hospital in Istanbul, Turkey. Participants enrolled in the study were selected among patients admitted to the cardiology outpatient clinic from January 2012 to November 2013. The study population included 111 (mean age $47 \pm 9,49.5 \%$ male) consecutive patients with MetS and 96 (mean age $45 \pm 9,58.3 \%$ male) control subjects without MetS.

The exclusion criteria of the present study were defined as follows: angina, acute coronary syndrome, heart failure history, congenital, pericardial and valvular heart disease, atrial fibrillation or flutter, secondary hypertension, renal disease, thyroid disorders, malignancies, chronic obstructive pulmonary disease, pulmonary hypertension, atrioventricular conduction abnormality, any QRS morphology with a QRS duration $120 \mathrm{~ms}$ or more (bundle branch block patterns; left, right bundle branch block and intraventricular conduction delay), segmental wall motion abnormalities, LV ejection fraction $(\mathrm{LVEF})<55 \%$, pregnancy, and inflammatory diseases. Written informed consent was obtained from all patients following approval of the study by the Institutional Review Board. The study was consistent with the Declaration of Helsinki.

\section{Study protocol}

Patients with MetS and control subjects without MetS were included in the study. The following demographic data were collected for all patients: age, sex, smoking status, body mass index (BMI), waist circumference, heart rate, and blood pressure. Blood samples were drawn following an overnight fasting period to measure fasting serum glucose, plasma lipids (i.e., triglyceride, HDL-C, total cholesterol, and low-density lipoprotein cholesterol [LDL-C] concentrations) and creatinine. Standard 12-lead ECGs were taken. Conventional echocardiography and tissue Doppler imaging were performed on all the subjects.

\section{Diagnosis and definitions}

The diagnosis of MetS was based on the presence of 3 or more of the risk factors for MetS established by the NCEP ATP III 2005 guidelines: systolic blood pressure (SBP) and diastolic blood pressure $(\mathrm{DBP}) \geq 130 / \geq 85 \mathrm{~mm} \mathrm{Hg}$, fasting plasma glucose $\geq 100 \mathrm{mg} / \mathrm{dL}$, waist circumference $>102 \mathrm{~cm}$ for men and $>88 \mathrm{~cm}$ for women, fasting triglycerides $>150 \mathrm{mg} / \mathrm{dL}$, and HDL-C $<40 \mathrm{mg} / \mathrm{dL}$ for men and $<50 \mathrm{mg} / \mathrm{dL}$ for women [2]. Diagnosis of diabetes was based on the criteria of the World Health Organization published in 2006 [11], and arterial hypertension was based on the recommendations of the European Society of Cardiology Hypertension Guideline, published in 2007 [12].

\section{Electrocardiogram}

Standard 12-lead surface resting ECGs (filter range, $0.5-150 \mathrm{~Hz}, 25 \mathrm{~mm} / \mathrm{s}, 10 \mathrm{~mm} / \mathrm{mV}$ ) were recorded for all the patients. These ECGs were evaluated by 2 cardiologists blinded to the patient data. Fragmented QRS was defined by the presence of various RSR' patterns (QRS duration $<120 \mathrm{~ms}$ ) with or without $\mathrm{Q}$ wave, which includes an additional $R$ wave ( $R^{\prime}$ ) or notching of the $\mathrm{R}$ wave or $\mathrm{S}$ wave, or the presence of more than one R' fragmentation without typical bundle branch block in 2 contiguous leads corresponding to a major coronary artery territory [8]. The standard 12-lead ECG was analyzed without using any magnification.

Conventional echocardiographic examination

All transthoracic echocardiographic examinations were performed with the GE Vivid S6 
Vingmed System 5 (Norway, Horten), which is equipped with $2.5-4 \mathrm{MHz}$ transducers. All patients were examined in the left lateral and supine positions with 2 dimensional, M-mode, pulsed, and tissue Doppler echocardiography. Single-lead ECG recordings were obtained continuously. For all measurements, the average of at least 5 cardiac cycles was used.

M-mode measurements and conventional Doppler echocardiographic examinations were performed based on the criteria of the American Society of Echocardiography and European Society of Echocardiography guidelines [13]. LV end-systolic and end-diastolic dimensions were measured in the parasternal long-axis views. LVEF was estimated by Simpson's rule. Left atrial maximum volume was measured in apical 4-chamber view at end-diastole. The mitral inflow peak velocity during early filling (E) and late filling from atrial contraction (A) were measured. The LV mass was calculated using the formula as previously described [14]. LV mass index (LVMI) was indexed for the surface area.

\section{Tissue Doppler imaging}

Doppler tissue echocardiography was performed using transducer frequencies between 3.5 and $4.0 \mathrm{MHz}$ by adjusting the spectral-pulsed Doppler signal filters until a Nyquist limit of 15 to $20 \mathrm{~cm} / \mathrm{s}$ was reached, and then using the minimal optimal gain. Five consecutive cycles were recorded using a frame rate greater than $150 \mathrm{fps}$. The monitor sweep speed was set at 50 to $100 \mathrm{~mm} / \mathrm{s}$ to optimize the spectral display of myocardial velocities. Every effort was made to align the pulsed-wave cursor to ensure that the Doppler angle of incidence was as close to 0 as possible to the direction of the walls. In the apical 4-chamber view, the pulsed Doppler sample volume was placed at the level of the LV mitral annulus end expiration [15].

The peak myocardial velocity during isovolumic contraction (IVV), acceleration time (AT) of peak myocardial velocity during isovolumic contraction, peak myocardial systolic velocity (Sa), peak early and late diastolic velocities (E' and A'), isovolumic contraction time (IVCT), isovolumic relaxation time (IVRT), and ejection time (ET) were measured. The E/E' ratio was calculated. The myocardial performance index (MPI) was calculated as the sum of the IVCT and the IVRT divided by the ET. The isovolumic acceleration (IVA) was defined as the ratio of IVV divided by the AT. All measurements were obtained by a single observer who was blinded to the clinical details.

\section{Statistical analyses}

Statistical analyses were performed using the SPSS software version 17.0 for Windows (SPSS Inc., Chicago, Illinois, USA). The variables were investigated using visual (histograms, probability plots) and analytical methods (KolmogorovSmirnov/Shapiro-Wilk's test) to determine the normal distribution. Descriptive analyses are presented using means and standard deviation. The categorical variables are expressed as numbers and percentages. Numerical variables were compared using a Student's t-test or the Mann-Whitney $\mathrm{U}$ test. Categorical data were compared with the $\chi^{2}$ test. Spearman correlation coefficients were used to assess the relationship between continuous variables. Stepwise logistic univariate and multivariate regression analyses were conducted to identify significant determinants of $\mathrm{fQRS}$. A $\mathrm{p}$ value of less than 0.05 was considered significant.

\section{Results}

Analyses of the study population

Demographic, clinical, and laboratory parameters of patients and control groups are shown in Table 1 . There were no significant differences between two groups in terms of age, gender, smoking status, heart rate, serum creatinine, and total cholesterol levels. MetS patients had significantly higher SBP, DBP, waist circumference, triglycerides, BMI, LDL-C, and fasting plasma glucose level than the controls. HDL-C levels were lower in the MetS group than in the control group. Fragmented QRS was more common among MetS patients $(26.1 \%$ vs. $14.6 \%, \mathrm{p}=0.041)$.

LV end-systolic and diastolic diameters and LVEF were similar between the two groups. LVMI, interventricular septum (IVS), posterior wall (PW), and left atrial (LA) max volume were higher in patients with MetS. In terms of diastolic function, conventional Doppler parameters $\mathrm{E}$ and $\mathrm{A}$, tissue Doppler parameters E' and A', and E/E' ratio were significantly impaired in MetS patients. Although Sa and IVV were similar between the two groups, IVA was significantly reduced in patients with MetS. The MPI reflecting both systolic and diastolic functions was significantly higher in patients with MetS compared to the controls (Table 2).

\section{Subgroup analyses of MetS patients}

After finding that $\mathrm{fQRS}$ is more common among MetS patients, they were divided into two groups: the fQRS (+) group (29 patients) and fQRS (-) group (81 patients). Then we compared all the parameters between these groups. In fQRS 
Table 1. Demographic, clinic and laboratory parameters of patients with or without metabolic syndrome (MetS).

\begin{tabular}{lccc}
\hline & MetS- $(\mathbf{n}=96)$ & MetS+ $(\mathbf{n}=111)$ & P \\
\hline Age [years] & $45 \pm 9$ & $47 \pm 9$ & 0.090 \\
Sex, male & $56(58.3 \%)$ & $55(49.5 \%)$ & 0.206 \\
Smoking & $28(29.2 \%)$ & $32(28.8 \%)$ & 0.957 \\
Body mass index [kg/m $]$ & $27 \pm 4$ & $30 \pm 4$ & $<0.001$ \\
Waist circumference [cm] & $92 \pm 10$ & $103 \pm 10$ & $<0.001$ \\
Diastolic blood pressure [mm Hg] & $85 \pm 13$ & $94 \pm 11$ & $<0.001$ \\
Systolic blood pressure [mm Hg] & $138 \pm 21$ & $151 \pm 2$ & $<0.001$ \\
Heart rate [bpm] & $77 \pm 12$ & $77 \pm 13$ & 0.896 \\
Fasting plasma glucose [mg/dL] & $94 \pm 8$ & $109 \pm 9$ & $<0.001$ \\
HDL-C [mg/dL] & $54 \pm 17$ & $42 \pm 10$ & $<0.001$ \\
Triglyceride [mg/dL] & $114 \pm 67$ & $208 \pm 115$ & $<0.001$ \\
Total cholesterol [mg/dL] & $205 \pm 42$ & $214 \pm 41$ & 0.100 \\
LDL-C [mg/dL] & $129 \pm 39$ & $140 \pm 31$ & 0.033 \\
Creatinine [mg/dL] & $0.79 \pm 0.15$ & $0.79 \pm 0.16$ & 0.944 \\
Fragmented fORS & $14(14.6 \%)$ & $29(26.1 \%)$ & 0.041 \\
\hline
\end{tabular}

HDL-C — high density lipoprotein cholesterol; LDL-C — low density lipoprotein cholesterol

Table 2. Conventional and tissue Doppler imaging measurements of left ventricle.

\begin{tabular}{|c|c|c|c|}
\hline & MetS- $(n=96)$ & MetS $+(n=111)$ & $\mathbf{P}$ \\
\hline LVEDD [mm] & $48.2 \pm 4.2$ & $48.1 \pm 4.2$ & 0.850 \\
\hline LVESD [mm] & $29.6 \pm 4.0$ & $29.2 \pm 3.2$ & 0.462 \\
\hline Interventricular septum (mm) & $10.5 \pm 2.0$ & $11.6 \pm 1.9$ & $<0.001$ \\
\hline Posterior wall [mm] & $9.1 \pm 1.5$ & $9.9 \pm 1.5$ & $<0.001$ \\
\hline Ejection fraction [\%] & $67.5 \pm 5.2$ & $66.4 \pm 4.9$ & 0.142 \\
\hline LV mass index $\left[\mathrm{g} / \mathrm{m}^{2}\right]$ & $90.4 \pm 23.0$ & $101.1 \pm 23.8$ & 0.001 \\
\hline LA maximum volume $[\mathrm{mL}]$ & $47.8 \pm 13.8$ & $53.2 \pm 11.5$ & 0.003 \\
\hline E velocity $[\mathrm{cm} / \mathrm{s}]$ & $83.7 \pm 13.9$ & $79.3 \pm 14.3$ & 0.025 \\
\hline A velocity $[\mathrm{cm} / \mathrm{s}]$ & $70.0 \pm 16.1$ & $78.2 \pm 17.2$ & 0.001 \\
\hline $\mathrm{E}^{\prime}$ velocity $[\mathrm{cm} / \mathrm{s}]$ & $11.8 \pm 3.3$ & $9.7 \pm 2.4$ & $<0.001$ \\
\hline$A^{\prime}$ velocity $[\mathrm{cm} / \mathrm{s}]$ & $11.5 \pm 2.2$ & $12.5 \pm 1.9$ & 0.001 \\
\hline E/E' ratio & $7.6 \pm 2.9$ & $8.6 \pm 2.8$ & 0.018 \\
\hline Sa $[\mathrm{cm} / \mathrm{s}]$ & $9.4 \pm 1.5$ & $9.4 \pm 1.7$ & 0.764 \\
\hline IVV [cm/s] & $7.3 \pm 2.2$ & $7.7 \pm 2.1$ & 0.167 \\
\hline IVA $\left[\mathrm{m} / \mathrm{s}^{2}\right]$ & $2.9 \pm 0.9$ & $2.4 \pm 0.4$ & $<0.001$ \\
\hline MPI & $0.47 \pm 0.1$ & $0.5 \pm 0.1$ & 0.001 \\
\hline
\end{tabular}

MetS - metabolic syndrome; LV — left ventricular; LVEDD — left ventricular end diastolic diameter; LVESD — left ventricular end systolic diameter; $\mathrm{E}$ - mitral inflow peak early diastolic wave velocity; A - mitral inflow peak late diastolic wave velocity $\mathrm{E}^{\prime}-$ flow velocity of the early diastole using tissue Doppler echocardiography; $A^{\prime}$ - flow velocity of the late diastole using tissue Doppler echocardiography; Sa - peak velocity of myocardial systolic wave; IVV - isovolumic velocity; IVA - isovolumic acceleration; MPI — myocardial performance index

(+) MetS patients, LV end-systolic and diastolic diameters, LVEF, A, Sa, and IVV parameters were similar compared to fQRS (-) patients. In fQRS (+) MetS patients, LVMI, IVS, PW and LA max volume,
E, E/E', A', and MPI were higher, while E' and IVA were lower (Table 3).

The results of the correlation analyses are shown in Table 4. Fragmented QRS was positively 
Table 3. Echocardiographic parameters of metabolic syndrome patients with and without fragmented QRS (fQRS).

\begin{tabular}{|c|c|c|c|}
\hline & fQRS- $(n=81)$ & fQRS $+(n=29)$ & $\mathbf{P}$ \\
\hline LVEDD [mm] & $47.7 \pm 3.7$ & $49.2 \pm 5.3$ & 0.151 \\
\hline LVESD [mm] & $28.6 \pm 2.9$ & $30.3 \pm 3.8$ & 0.077 \\
\hline Interventricular septum [mm] & $11.2 \pm 1.9$ & $12.7 \pm 1.5$ & $<0.001$ \\
\hline Posterior wall [mm] & $9.7 \pm 1.5$ & $10.6 \pm 1.6$ & 0.001 \\
\hline Ejection fraction [\%] & $66.3 \pm 5.1$ & $66.6 \pm 4.1$ & 0.873 \\
\hline LV mass index $\left[\mathrm{g} / \mathrm{m}^{2}\right]$ & $93.7 \pm 21.1$ & $121.0 \pm 18.6$ & $<0.001$ \\
\hline LA maximum volume & $51.4 \pm 11.7$ & $58.2 \pm 9.4$ & 0.005 \\
\hline E velocity $[\mathrm{cm} / \mathrm{s}]$ & $76.3 \pm 12.7$ & $87.6 \pm 15.4$ & $<0.001$ \\
\hline A velocity $[\mathrm{cm} / \mathrm{s}]$ & $78.7 \pm 18.3$ & $76.7 \pm 13.0$ & 0.589 \\
\hline$E^{\prime}$ velocity $[\mathrm{cm} / \mathrm{s}]$ & $10.3 \pm 2.5$ & $8.0 \pm 1.1$ & $<0.001$ \\
\hline$A^{\prime}$ velocity $[\mathrm{cm} / \mathrm{s}]$ & $12.2 \pm 2.0$ & $13.1 \pm 1.4$ & 0.023 \\
\hline E/E' ratio & $7.6 \pm 2.4$ & $11.5 \pm 2.0$ & $<0.001$ \\
\hline $\mathrm{Sa}[\mathrm{cm} / \mathrm{s}]$ & $9.5 \pm 1.8$ & $9.0 \pm 1.3$ & 0.179 \\
\hline IVV $[\mathrm{cm} / \mathrm{s}]$ & $7.8 \pm 2.1$ & $7.7 \pm 1.9$ & 0.805 \\
\hline IVA $\left[\mathrm{m} / \mathrm{s}^{2}\right]$ & $2.5 \pm 0.45$ & $2.0 \pm 0.32$ & $<0.001$ \\
\hline MPI & $0.51 \pm 0.08$ & $0.59 \pm 0.07$ & $<0.001$ \\
\hline
\end{tabular}

LV — left ventricular; LVEDD — left ventricular end diastolic diameter; LVESD — left ventricular end systolic diameter; IVS - interventricular septum; PW - posterior wall; LA max volume - left atrium maximum volume; E - mitral inflow peak early diastolic wave velocity; A mitral inflow peak late diastolic wave velocity $E^{\prime}$ - flow velocity of the early diastole using tissue Doppler echocardiography; $A^{\prime}$ - flow velocity of the late diastole using tissue Doppler echocardiography; Sa - peak velocity of myocardial systolic wave; IVV — isovolumic velocity; IVA - isovolumic acceleration; MPI — myocardial performance index

correlated with DBP and SBP, waist circumference, triglyceride levels, IVS, PW, LV mass index, LA max volume, E velocity, A' velocity, E/E' ratio, and MPI. HDL levels, E' velocity, and IVA were inversely correlated with $\mathrm{fQRS}$. There was no correlation between $\mathrm{fQRS}$ and age, fasting plasma glucose levels, number of risk factors, A velocity, $\mathrm{Sa}$, or BMI.

In univariate analysis, to predict the presence of fQRS in MetS patients, DBP and SBP, HDL and triglyceride levels, E/E' ratio, LV mass index, LA max volume, and IVA were found to be parameters associated with $\mathrm{fQRS}$ (Table 5). After adjustment for potential confounders, fQRS was associated with $\mathrm{E} / \mathrm{E}$ ' ratio and IVA in multivariate logistic regression analysis.

\section{Discussion}

This study demonstrated that: 1) fQRS is more common in MetS patients; 2) MetS is associated with subclinical LV systolic dysfunction and LV diastolic dysfunction; 3 ) in subgroup analyses of MetS patients, the presence of $\mathrm{AQRS}$ on ECG was associated with more prominent subclinical LV systolic and LV diastolic dysfunctions; 4) the presence of $\mathrm{fQRS}$ is correlated with MetS components and LV systolic and diastolic parameters; and 5) E/E' ratio and IVA were independent predictors of $\mathrm{fQRS}$ presence in patients with MetS.

Metabolic syndrome is an escalating publichealth problem. The prevalence of MetS in industrialized countries is about $22 \%$ of the adult population and over $40 \%$ of those aged 50 and older [16]. MetS is associated with a 5-fold increase in the risk of type 2 diabetes mellitus and 2 -fold risk of developing cardiovascular disease over the next 5 to 10 years [17]. MetS is known to be associated with deterioration in LV systolic and diastolic functions [3-5]. Myocardial fibrosis is thought to be one of the contributors to this deterioration $[6,7,18]$. Myocardial fibrosis can be diagnosed histopathologically and with the help of cardiac magnetic resonance imaging and scintigraphic methods $[19,20]$. These methods are not readily available and are rather expensive.

Fragmented QRS on ECG has been found a marker of myocardial scarring or fibrosis in various diseases $[8,21,22]$. It can serve as an inexpensive and readily available electrocardiographic index of myocardial fibrosis. The prevalence of $\mathrm{fQRS}$ was found to be $9.2 \%$ among healthy 
Table 4. Correlation between presence of fragmented QRS with demographic and echocardiographic parameters in patients with metabolic syndrome.

\begin{tabular}{lcc}
\hline & $\mathbf{R}$ & $\mathbf{p}$ \\
\hline Age & -0.132 & 0.167 \\
Sex & 0.190 & 0.046 \\
Waist circumference & 0.208 & 0.029 \\
Diastolic blood pressure & 0.256 & 0.007 \\
Systolic blood pressure & 0.310 & 0.001 \\
Fasting plasma glucose & -0.069 & 0.475 \\
Triglyceride & 0.360 & $<0.001$ \\
HDL-C & -0.175 & 0.066 \\
Number of risk factors & 0.014 & 0.886 \\
E velocity & 0.325 & 0.001 \\
A velocity & -0.220 & 0.815 \\
E' velocity & -0.429 & $<0.001$ \\
A' velocity & 0.196 & 0.039 \\
Sa & -0.114 & 0.234 \\
MPI & 0.416 & $<0.001$ \\
Isovolumic acceleration & -0.493 & $<0.001$ \\
LV mass index & 0.523 & $<0.001$ \\
Interventricular septum & 0.365 & $<0.001$ \\
Posterior wall & 0.225 & 0.018 \\
Body mass index & 0.014 & 0.881 \\
E/E' ratio & 0.687 & $<0.001$ \\
LV maximum volume & 0.273 & 0.004 \\
\hline
\end{tabular}

HDL-C - high density lipoprotein cholesterol; E - mitral inflow peak early diastolic wave velocity; A - mitral inflow peak late diastolic wave velocity $E^{\prime}$ - flow velocity of the early diastole using tissue Doppler echocardiography; $A^{\prime}$ - flow velocity of the late diastole using tissue Doppler echocardiography; Sa - peak velocity of myocardial systolic wave; $\mathrm{MPI}$ - myocardial performance index; LV — left ventricle middle-aged Finnish subjects [23]. The prevalence is increased in various diseases, including ST elevation myocardial infarction (21.9\%) [7], cardiac sarcoidosis (75\%) [21], and chronic kidney disease (60\%) [22]. These data suggest that fQRS prevalence increases with myocardial involvement. We found that the prevalence of $\mathrm{fQRS}$ is higher in MetS compared to non-MetS controls $(26.1 \%$ vs. $14.6 \%$, $\mathrm{p}=0.041$ ). Our control group was chosen from the cardiology outpatient clinic, and some patients had 1 or 2 aspects of MetS. Our control group's fQRS percentage can be expected to be higher than that of the healthy population.

Metabolic syndrome is known to be associated with deterioration in LV systolic and diastolic functions [3-5]. Consistent with previous studies, in our study, MetS patients had diastolic dysfunction detected by lower $\mathrm{E}$ velocity and $\mathrm{E}$ ' velocity and higher A velocity, A' velocity, E/E' ratio than the non-MetS controls. LVEF and Sa were not different, but tissue Doppler parameter IVA was lower in MetS patients, indicating LV subclinical systolic dysfunction. IVA reflects the acceleration of the myocardium at the very beginning of the isovolumic contraction period. IVA remains unaffected by the changes in the preload and afterload within the physiological range [24]. It can detect even small changes in the contractile function and is well correlated with the invasive or noninvasive measures of $\mathrm{LV} \mathrm{dp/dt}[24,25]$. This parameter has been successfully validated in clinical studies [26, 27].

There is no data as to whether the presence of $\mathrm{fQRS}$ predicts pronounced LV systolic and diastolic dysfunction in MetS patients. In our study, the presence of $\mathrm{fQRS}$ on ECG in MetS patients was

Table 5. Logistic regression analysis of fragmented QRS for both metabolic syndrome parameters and echocardiographic parameters in metabolic syndrome patients.

\begin{tabular}{lcccccc}
\hline & Unadjusted & & & \multicolumn{2}{c}{ Adjusted } \\
\cline { 2 - 3 } \cline { 5 - 6 } & Odds ratio (95\% CI) & $\mathbf{P}$ & & Odds ratio (95\% CI) & $\mathbf{P}$ \\
\hline Waist circumference & $1.04(0.99-1.09)$ & & 0.056 & & $1.04(0.95-1.13)$ & 0.399 \\
Diastolic blood pressure & $1.04(1.00-1.09)$ & & 0.038 & & $0.97(0.85-1.09)$ & 0.970 \\
HDL-C & $0.94(0.89-0.99)$ & & 0.033 & & $0.94(0.85-1.02)$ & 0.936 \\
Triglyceride & $1.00(1.00-1.01)$ & & 0.006 & & $1.01(0.99-1.02)$ & 0.068 \\
Systolic blood pressure & $1.04(1.01-.06)$ & & 0.001 & & $1.07(0.99-1.14)$ & 0.058 \\
Fasting plasma glucose & $0.99(0.98-1.01)$ & & 0.755 & & & \\
Risk factor number & $1.09(0.59-2.01)$ & & 0.772 & & & \\
E/E' ratio & $2.19(1.58-3.03)$ & & $<.001$ & & $1.53(1.05-2.22)$ & 0.024 \\
LV mass index & $1.06(1.03-1.10)$ & $<0.001$ & & $1.05(1.00-1.11)$ & 0.048 \\
LA maximum volume & $1.05(1.01-1.09)$ & & 0.008 & & $1.04(0.95-1.13)$ & 0.399 \\
Isovolumic acceleration & $0.04(0.01-0.17)$ & $<0.001$ & & $0.01(0.01-0.26)$ & 0.007 \\
\hline
\end{tabular}

$\mathrm{Cl}$ - confidence interval; HDL-C — high density lipoprotein cholesterol; $\mathrm{E}$ - mitral inflow peak early diastolic wave velocity; $\mathrm{E}^{\prime}$ - flow velocity of the early diastole using tissue Doppler echocardiography; LA - left atrium 
associated with pronounced diastolic dysfunction expressed by higher $\mathrm{E} / \mathrm{E}$ ' ratio and lower $\mathrm{E}$ ' velocity. A novel tissue Doppler parameter IVA was lower in the fQRS-positive group, indicating subclinical LV systolic dysfunction. In light of these results, we think that the presence of $\mathrm{fQRS}$ on ECG is an indicator of pronounced LV systolic and diastolic dysfunction in MetS patients.

Metabolic syndrome consists of 5 metabolic and physiological risk factors. Particular components of MetS - hypertension, diabetes, and obesity - have been found to be associated with myocardial fibrosis [28-30]. Kosmala et al. [7] used surrogate serological markers of fibrotic processes and myocardial deformation parameters, and showed that subclinical LV systolic dysfunction and diastolic dysfunction is associated with a high degree of fibrosis in MetS patients. In our study, the presence of $\mathrm{fQRS}$ was correlated with waist circumference, SBP and DBP, and triglyceride level. In univariate logistic regression analysis, the MetS parameters SBP and DBP, HDL, and triglyceride levels were associated with the presence of $\mathrm{fQRS}$. In multivariate logistic regression analysis, only LV IVA and $\mathrm{E} / \mathrm{E}$ ' ratio were independent determinants of the presence of $\mathrm{fQRS}$ on ECG. In light of these data, we think that myocardial fibrosis occurs with the contribution of particular MetS components, and causes the deterioration of LV functions. Myocardial fibrosis causing pronounced dysfunction can be predicted by the presence of $\mathrm{fQRS}$ on ECG.

\section{Limitations of the study}

This study has several limitations. The first one is small sample size. Secondly, we did not assess prospectively the effect of the presence of fQRS on mortality and morbidity. Thirdly, we did not show cardiac fibrosis histopathologically or with other imaging modalities. Finally, coronary artery disease was excluded based on history, electrocardiography, or echocardiography (wall motion abnormality), and we did not perform exercise stress tests or coronary angiography.

\section{Conclusions}

Fragmented QRS is more common in MetS patients than in non-MetS patients. The presence of $\mathrm{fQRS}$ is associated with pronounced subclinical LV systolic dysfunction and diastolic dysfunction in MetS patients.

Conflict of interest: None declared

\section{References}

1. Eckel RH, Grundy SM, Zimmet PZ. The metabolic syndrome. Lancet, 2005; 365: 1415-1418.

2. Grundy SM, Cleeman JI, Daniels SR et al. American Heart Association; National Heart, Lung and Blood Institute Scientific Statement. Circulation, 2005; 112: 2735-2732.

3. Erturk M, Oner E, Kalkan AK et al. Anatolian J Cardiol, 2015; 15: 42-49.

4. Ivanovic B, Tadic M, Simic D. Are all criteria of metabolic syndrome equally harmful? Acta Cardiol, 2011; 66: 189-196.

5. Crendal E, Walther G, Vinet A et al. Myocardial deformation and twist mechanics in adults with metabolic syndrome: Impact of cumulative metabolic burden. Obesity (Silver Spring), 2013; 21: 679-686.

6. Sciarreta S, Ferrucci A, Ciavarella GM et al. Markers of inflammation and fibrosis are related to cardiovascular damage in hypertensive patients with metabolic syndrome. Am J Hypertens, 2007; 20: 784-791.

7. Kosmala W, Przewlocka-Kosmala M, Wojnalowicz A, Mysiak A, Marwick TH Integrated backscatter as a fibrosis marker in the metabolic syndrome: Association with biochemical evidence of fibrosis and left ventricular dysfunction. Eur Heart J Cardiovasc Imag, 2012; 13: 459-467.

8. Das MK, Khan B, Jacob S, Kumar A, Mahentiran J. Significance of a fragmented QRS complex versus a $Q$ wave in patients with coronary artery disease. Circulation, 2006; 113: 2495-2501.

9. Das MK, Michael MA, Suradi H et al. Usefulness of fragmented QRS on a 12-lead electrocardiogram in acute coronary syndrome for predicting mortality. Am J Cardiol, 2009; 104: 1631-1637.

10. Akgul O, Uyarel H, Pusuroglu O et al. Predictive value of a fragmented QRS complex in patients undergoing primary angioplasty for ST elevation myocardial infarction. Ann Noninvasive Electrocardiol, 2015; 20: 263-272. doi: 10.1111/anec.12179.

11. Who Guideline Development Committee. Definition and diagnosis of diabetes mellitus and intermediate hyperglycemia. Report of a WHO/IDF Consultation, Geneve 2006.

12. Mancia G, De Backer G, Dominiczak A et al. 2007 ESH-ESC Practice Guidelines for the Management of Arterial Hypertension: ESH-ESC Task Force on the Management of Arterial Hypertension. J Hypertens, 2007; 25: 1751-1762.

13. Lang RM, Biering M, Devereux RB et al. Recommendations for chamber quantification: A report from the American society of echocardiography's guidelines and standards committee and the chamber quantification writing group, developed in conjunction with the European association of echocardiography, a branch of the European society of cardiology. J Am Soc Echocardiogr, 2005; 18: 1440-1463.

14. Devereux RB, Alonso DR, Lutas EM et al. Echocardiographic assessment of left ventricular hypertrophy: comparison to necropsy finding. Am J Cardiol, 1986; 57: 450-458.

15. Quinones MA, Otto CM, Stoddard M, Waggoner A, Zoghbi WA. Recommendations for quantification of Doppler Echocardiography: A report from the Doppler Quantification task force of the nomenclature and standards committee of the American Society of Echocardiography. J Am Soc Echocardiogr, 2002; 15: 167-184.

16. Ford ES, Giles WH, Dietz WH. Prevalence of the metabolic syndrome among US adults: findings from the third National Health and Nutrition Examination Survey. JAMA, 2002; 287: 356-359.

17. Alberti KG, Eckel RH, Grundy SM et al. Harmonizing the metabolic syndrome: a joint interim statement of the International Diabetes Federation Task Force on Epidemiology and Preven- 
Cardiology Journal 2015, Vol. 22, No. 6

tion; National Heart, Lung, and Blood Institute; American Heart Association; World Heart Federation; International Atherosclerosis Society; and International Association for the Study of Obesity. Circulation, 2009; 120: 1640-1645.

18. Kosmala W, Kosmala-Przewlocka M, Osadnik-Szczepanik H, Mysiak A, O'Moore-Sullivan T, Marwick TH. A randomized study of aldosterone antagonism on LV function, structure, and fibrosis markers in metabolic syndrome. J Am Coll Cardiol Imag, 2011; 4: 1239-1249.

19. Anderson Kr, Sutton MG, Lie JT. Histopathological types of cardiac fibrosis in myocardial disease. J Pathol, 1979; 128: 79-85.

20. Ferrari VA, Witschey WR, Zhou R. Cardiac magnetic resonance assessment of myocardial fibrosis: honing new clinical tools. Circ Cardiovasc Imag, 2011; 4: 604-606.

21. Sculler JL, Olson MD, Zipse MM et al. Electrocardiographic characteristics in patients with pulmonary sarcoidosis indicating cardiac involvement. J Cardiovasc Electrophysiol, 2011; 22: 1243-1248.

22. Adar A, Kiris A, Ulusoy S et al. Fragmented QRS in associated with subclinical left ventricular dysfunction in patients with chronic kidney disease. Acta Cardiol, 2014; 69: 385-390.

23. Terho HK, Tikkanen JT, Juntilla JM et al. Prevalence and prognostic significance of fragmented QRS complex in middle-aged subjects with and without clinical or electrocardiographic evidence of cardiac disease. Am J Cardiol, 2014; 114: 141-147. doi: 10.1016/j.amjcard.2014.03.066.
24. Vogel M, Schmidt MR, Kristiansen SB et al. Validation of myocardial acceleration during isovolumic contraction as a novel noninvasive index of right ventricular contractility. Comparison with ventricular pressure-volume relations in an animal model. Circulation, 2002; 105: 1693-1699.

25. Hashimoto I, Li X-K, Bhat AH Jones M, Sahn DJ. Quantitative assessment of regional peak myocardial acceleration during isovolumic contraction and relaxation times by tissue Doppler imaging. Heart, 2005; 91: 811-816.

26. Erturk M, Aksu HU, Celik O et al. Evaluation of the effect of mitral stenosis severity on the left ventricular systolic function using isovolumic myocardial acceleration. Cardiol J, 2014; 21: 442-448.

27. Erturk M, Buturak A, Pusuroglu $\mathrm{H}$ et al. Comparison of subclinical left and right ventricular systolic dysfunction in non-dipper and dipper hypertensives: Impact of isovolumic acceleration. Clin Exp Hypertens, 2014; 36: 572-578.

28. Gonzales A, Lopez B, Diez J. Fibrosis in hypertensive heart disease role of the angiotensin-aldesteron system. Med Clin N Am, 2004; 88: 93-97.

29. Adeghate E, Singh J. Structural changes in myocardium during diabetes-induced cardiomyopathy. Heart Fail Rev, 2014; 19: 15-23.

30. Kosmala W, Kosmala-Przewlocka M, Osadnik-Szczepanik H, Mysiak A, Marwick TH. Fibrosis and cardiac function in obesity: A randomized controlled trial of aldosterone blockade. Heart, 2013; 99: 320-326. 